\title{
Rash and neutropenia after the administration of oxaliplatin and 5-fluorouracil plus calcium folinate injection: a case report
}

\author{
Jiwei Huang" ${ }^{\#}$, Caiyun Jiang", Zhuying Liu, Weifeng Zheng, Ximei Zhu, Zulin Huang \\ Department of Pharmacy, Third Affiliated Hospital of Sun Yat-sen University, Guangzhou, China \\ \#These authors contributed equally to this work. \\ Correspondence to: Jiwei Huang. Department of Pharmacy, Third Affiliated Hospital of Sun Yat-sen University, Guangzhou 510630, China. \\ Email: $947543512 @ q q . c o m$.
}

\begin{abstract}
A 56-year-old male patient was admitted due to a "rectal malignant tumor". He suffered from rash and neutropenia after multiple chemotherapy sessions including oxaliplatin, 5-fluorouracil (5FU), and calcium folinate injection (CF) which are called FOLFOX regimen for short. The rash was treated with methylprednisolone + promethazine + calcium gluconate, and the neutropenia was treated by subcutaneous injection of the Recombinant Human Granulocyte Colony-Stimulating Factor Injection, the symptoms were relieved. Moreover, rashes and neutropenia are known common adverse reactions after intravenous administration of FOLFOX regimen. Based on the patient's symptoms and the timing of drug administration, a diagnosis of "rash and neutropenia due to the use of FOLFOX regimen" was made. Oxaliplatin and CF may also cause allergic reactions, including skin erythema and anaphylactic shock, etc. Once allergic reaction occurs, the fatality rate is higher than that of Penicillin. Therefore, sufficient attention should be paid to the patients reported in this paper who received FOLFOX regimen for multiple times and had multiple rashes and adverse reactions of neutropenia. Medical staff should closely monitored the adverse reactions and changes in vital signs of patients treated with this regimen during chemotherapy, and the chemotherapy regimen should be adjusted or terminated when necessary. The adverse reactions reported in this article deserve clinical attention.
\end{abstract}

Keywords: Oxaliplatin; 5-fluorouracil (5-FU); calcium folinate injection (CF); rash; neutropenia; case report

Submitted May 29, 2020. Accepted for publication Aug 21, 2020.

doi: 10.21037/apm-20-1321

View this article at: http://dx.doi.org/10.21037/apm-20-1321

\section{Introduction}

Colorectal cancer (CRC) is the second leading cause of cancer death in the United States (1). In China, its incidence and mortality has been also on the rise over recent decades, and now CRC ranks fourth in prevalence $(2,3)$. Most CRC cases are diagnosed at advanced stages (4), and the prognosis of CRC is poor (5). For patients with early and mid-term colon cancer, surgery or endoscopic treatment is the first choice. Surgery is the only cure for localized CRC. Adjuvant chemotherapy can be given according to the patient's condition after operation. For patients with early and mid-term CRC, surgery or endoscopic treatment is the first choice. Surgery is the only cure for localized colon cancer. Adjuvant chemotherapy can be given according to the patient's condition after operation. For patients who have undergone potentially curable colon cancer resection, postoperative adjuvant chemotherapy is used to eradicate micrometastases, thereby reducing the risk of recurrence and increasing the cure rate. For locally advanced primary colon cancer, multiple organ resection should be performed if it is potentially resectable. Neoadjuvant chemoradiotherapy combined with chemotherapy is a

$\wedge$ ORCID: 0000-0002-7647-5155. 
common method for the treatment of locally advanced rectal cancer. All planned systemic chemotherapy plus chemoradiotherapy should be implemented before operation, so as to make more patients achieve curative resection after chemotherapy and downgrade the stage of rectal cancer.

For patients with metastasis or postoperative recurrence, chemotherapy remains one of the mainstays of CRC treatment $(6,7)$ as it can effectively increase the long-term survival and postoperative survival rate of CRC patients. The FOLFOX regimen can extend the lives of CRC patients (8). In recent years, FOLFOX regimen has been widely used as the first-line treatment for metastatic CRC (9) and as the adjuvant therapy for stage III (Duke's C) CRC after complete resection of the primary tumor. Also, it has been recommended by U.S. National Comprehensive Cancer Network (NCCN), the European Society for Medical Oncology (ESMO), and the Chinese Society of Clinical Oncology (CSCO) as the preferred regimen for the adjuvant chemotherapy for stage III CRC and the chemotherapy of choice for advanced CRC. Chemotherapy may cause significant toxicity, and the main side effects of oxaliplatin are gastrointestinal reaction, neutropenia, thrombocytopenia and peripheral sensory neuropathy. Allergy symptoms should be monitored in patients with a history of platinum allergy. 5-fluorouracil (5-FU) combined with calcium folinate (CF) may lead to leukopenia or thrombocytopenia, gastrointestinal reactions, bleeding at the injection site, rash, pigmentation, etc. Serious adverse reactions include central neurotoxicity and myocardial ischemia. Blood cell count should be monitored regularly during medication. Fortunately, most of these symptoms are reversible after discontinuation of chemotherapy, and delayed and long-term effects are relatively rare, but oxaliplatin associated peripheral neuropathy may persist (10). Aranda et al. (11) and Pfeiffer et al. (12) reported the first use of cetuximab in combination with FOLFOX regimen for metastatic colorectal cancer, with cetuximab used for maintenance therapy. During the treatment regimen, adverse reactions including rash and neutropenia would occur. In this paper, we report a patient who received multiple FOLFOX chemotherapy regimens and had multiple adverse reactions of rash and neutropenia. In this case, the pretreatment with glucocorticoid dexamethasone sodium phosphate injection of $10 \mathrm{mg}$ before chemotherapy could not prevent the occurrence of allergic reactions. The adverse reactions reported in this paper warrant clinical attention and can be a reference for better service of patients.

We present the following article in accordance with the CARE reporting checklist (available at http://dx.doi. org/10.21037/apm-20-1321).

\section{Case presentation}

The patient was a 56-year-old male, who had undergone brain tumor surgery 10 years prior to being admitted to our center. He had no history of chronic disease (e.g., hypertension, diabetes, or coronary heart disease) or chronic infectious diseases (e.g., hepatitis or tuberculosis). Also, he denied any history of food and drug allergies, blood transfusion, or off-schedule vaccination. Half a year previous, he experienced excessive stools (which were occasionally accompanied by bloody stools) without any obvious cause. However, since there was no abdominal pain, bloating, diarrhea or discomfort such as headache, dizziness, nausea, or vomiting, he paid no attention to this condition, and the symptoms repeatedly occurred. He then visited a local county-level traditional Chinese medicine hospital, where colonoscopy revealed a rectal mass, which was pathologically confirmed as moderately differentiated adenocarcinoma. $\mathrm{He}$ presented to our outpatient department for further diagnosis and treatment and was admitted into the Department of Gastrointestinal Surgery due to "rectal malignant tumor". Adjuvant FOLFOX was applied in our department in 2019 on July 20, August 6, August 21, and October 8. The chemotherapy went smoothly, and the patient showed no obvious discomfort. On September 9, 2019, the patient successfully underwent laparoscopic Miles operation for rectal cancer under general anesthesia. On October 30, 2019 , the patient was re-admitted to receive neoadjuvant chemotherapy. He received further examinations and tests at re-admission. Routine blood test showed the following: absolute monocyte count, $0.67 \times 10^{9}$ cells $/ \mathrm{L}$; total white blood cell (WBC) count, $3.62 \times 10^{9}$ cells/L; absolute neutrophil count, $1.040 \times 10^{9}$ cells $/ \mathrm{L}$; and percentage of neutrophils, 0.2880 . Three lung tumor markers of CA199, liver function, and biochemical findings showed no abnormality. A second routine blood test (absolute monocyte count, $1.48 \times 10^{9}$ cells $/ \mathrm{L}$; total WBC count, $11.83 \times 10^{9}$ cells $/ \mathrm{L}$; absolute neutrophil count, $7.81 \times 10^{9}$ cells/L; and percentage of neutrophils, 0.6610 ) showed no obvious abnormality after the patient was administered with Recombinant Human Granulocyte Colony-Stimulating Factor Injection $75 \mu \mathrm{g}$ subcutaneous injection. The relevant contraindications to chemotherapy were ruled out. On October 31, 2019, 
dexamethasone sodium phosphate injection $10 \mathrm{mg}$ was injected intravenously 30 minutes before chemotherapy. The patient was treated with a FOLFOX regimen [oxaliplatin, $135 \mathrm{mg}$, CF, $0.6 \mathrm{~g}$, ivgtt; and $5-\mathrm{FU}, 0.6 \mathrm{~g}$ as a bolus, followed by a $3.8 \mathrm{~g}-5$-FU continuous infusion for 24 hours]. Severe skin itching and red rashes occurred during the chemotherapy. After intravenous injection of $40 \mathrm{mg}$ of methylprednisolone, the itchy and red rashes were remarkably improved. After another chemotherapy session with oxaliplatin produced by another manufacturer, the systemic itching recurred, which was alleviated again after intravenous injection of $40 \mathrm{mg}$ of Methylprednisolone. After chemotherapy, the patient's condition was stable. Routine blood test showed the following: absolute monocyte count, $0.58 \times 10^{9}$ cells $/ \mathrm{L}$; WBC count, $5.43 \times 10^{9}$ cells $/ \mathrm{L}$; absolute neutrophil count, $3.16 \times 10^{9}$ cells/L; and percentage of neutrophils, 0.582 . There was no obvious abnormality in liver function and biochemistry.

On November 24, 2019, the patient was re-admitted, and relevant examinations and tests were undertaken. Routine blood test showed that the absolute neutrophil count was $1.590 \times 10^{9}$ cells $/ \mathrm{L}$, the hemoglobin concentration was $128.000 \mathrm{~g} / \mathrm{L}$, and the percentage of neutrophils was 0.2860. Liver function test, biochemistry, and testing for tumor markers showed no obvious abnormality. After subcutaneous injection of the Recombinant Human Granulocyte Colony-Stimulating Factor Injection $75 \mu \mathrm{g}$, the patient was treated with oxaliplatin, $135 \mathrm{mg}$, ivgtt; $\mathrm{CF}, 0.6 \mathrm{~g}$, ivgtt; and 5-FU, $0.6 \mathrm{~g}$ as a bolus, followed by a $3.8 \mathrm{~g}-5-\mathrm{FU}$ ivgtt as postoperative adjuvant chemotherapy. Dexamethasone sodium phosphate injection $10 \mathrm{mg}$ was injected intravenously 30 minutes before chemotherapy. During the chemotherapy, there were scattered rashes with itching, but there was no discomfort such as dyspnea, nausea, or vomiting. His blood pressure was $114 / 44 \mathrm{mmHg}$, and his heart rate was 66 beats/min. The infusion of oxaliplatin was suspended, and intravenous injection of $40 \mathrm{mg}$ of Methylprednisolone and intramuscular injection of $25 \mathrm{mg}$ of Promethazine Hydrochloride were performed immediately. After the patient's symptoms were improved, the chemotherapy continued, and no abnormalities were seen afterwards. A routine blood test was conducted after chemotherapy that showed the following: absolute monocyte count, $0.6 \times 10^{9}$ cells $/ \mathrm{L}$; total $\mathrm{WBC}$ count, $16.14 \times 10^{9}$ cells $/ \mathrm{L}$; absolute neutrophil count, $13.49 \times 10^{9}$ cells/L, and percentage of neutrophils, 0.835. CA19-9, alpha fetoprotein, various vitamins, carcinoembryonic antigen, liver function, and biochemistry were not significantly abnormal.

On December 14, 2019, routine blood test showed that the absolute neutrophil count was $0.590 \times 10^{9}$ cells $/ \mathrm{L}$, the total red blood cell (RBC) count was $3.77 \times 10^{12}$ cells/L, hemoglobin concentration was $115.000 \mathrm{~g} / \mathrm{L}$, and the percentage of neutrophils was 0.1420 . Liver function test, biochemistry, and testing for tumor markers showed no obvious abnormality. After subcutaneous injection of the Recombinant Human Granulocyte Colony-Stimulating Factor Injection $75 \mu \mathrm{g}$ in 2019 on December 14, and December 16, routine blood test (absolute neutrophil count, $6.84 \times 10^{9}$ cells $/ \mathrm{L}$; the total $\mathrm{RBC}$ count was $3.96 \times 10^{12}$ cells $/ \mathrm{L}$, hemoglobin concentration was $118.000 \mathrm{~g} / \mathrm{L}$, and percentage of neutrophils, 0.5750 ) showed no obvious abnormalities. The patient's mental status and appetite were fair. The relevant contraindications to chemotherapy were ruled out. On December 17, 2019, dexamethasone sodium phosphate injection $10 \mathrm{mg}$ was injected intravenously 30 minutes before chemotherapy. The patient was treated with oxaliplatin, $120 \mathrm{mg}$, ivgtt; CF, $0.6 \mathrm{~g}$, ivgtt; and 5-FU, $0.6 \mathrm{~g}$ as a bolus, followed by a $3.8 \mathrm{~g}-5-\mathrm{FU}$ ivgtt as postoperative adjuvant chemotherapy. During the intravenous drip of oxaliplatin, the patient suffered from sudden systemic skin itching, which was accompanied by rashes. The infusion of chemotherapy drug was immediately suspended, and intravenous injection of $40 \mathrm{mg}$ of Methylprednisolone and intramuscular injection of $25 \mathrm{mg}$ of Promethazine Hydrochloride were performed immediately. The patient's symptoms were significantly relieved, and his vital signs were stable. The above chemotherapy regimen continued, and no obvious abnormal reactions were seen. A routine blood test was conducted after chemotherapy that showed the following: absolute monocyte count, $0.46 \times 10^{9}$ cells/L; total WBC count, $6.12 \times 10^{9}$ cells $/ \mathrm{L}$, absolute neutrophil count, $3.9 \times 10^{9}$ cells $/ \mathrm{L}$; and percentage of neutrophils, 0.637 .

The content involved in this case was in accordance with the ethical standards of the institutional and/or national research committee(s) and with the Helsinki Declaration (as revised in 2013). Written informed consent was obtained from the patient for publication of this study and any accompanying images.

\section{Discussion}

For patients with middle and advanced rectal cancer whose tumors are found to be difficult to resect or unresectable by preoperative evaluation, preoperative transforming 
chemotherapy should be given to achieve the goal of tumor reduction before surgery, so as to improve the surgical resection rate. In addition, neoadjuvant chemotherapy can also determine the response of the tumor to chemotherapy, which is helpful for postoperative planning. The patient was currently diagnosed as having a rectal malignant tumor, and according to the impact results, the clinical stage was T4NOMO, stage IIB. Considering that the tumor was large and adhered to the surrounding tissues, neoadjuvant chemotherapy was given first. The patients received adjuvant chemotherapy on July 20, 2019, August 6, 2019, and on August 21,2019. The chemotherapy process was smooth, and no obvious discomfort was observed. After chemotherapy, the tumor was reduced and "laparoscopic rectal cancer Miles" procedure was performed under general anesthesia on September 9, 2019. At present, the patient is returning to hospital for further chemotherapy.

Oxaliplatin is a non-cycle-specific anti-cancer drug. In the human body, oxaliplatin trans-1-diaminocyclohexane oxalate platinum II can be converted into bioactive monohydrate and dihydrate 1,2-diaminocyclohexane platinum derivatives after the replacement of unstable oxalate ligands by nonenzymatic reaction. These derivatives can form intra- and inter-chain cross-links with DNA and ultimately produce anti-tumor activity by inhibiting the synthesis of tumor cell DNA $(13,14)$. 5-FU can be converted to 5-fluoro-2deoxyuracil nucleotides, which has a potent inhibitory effect on thymidylate synthetase and can block the conversion of deoxyuridine monophosphate (dUMP) to deoxythymidine monophosphate (dTMP), ultimately inhibiting tumor cell DNA biosynthesis (15). The 5-FU/CF combination can effectively enhance the efficacy of 5-FU, and it is often used clinically to treat advanced rectal cancer and colon cancer (16).

Chemotherapy drugs mainly effect rapidly dividing tumor cells, but the selectivity is relatively weak, and they also have killing effect on normal cells with fast growth rate, including bone marrow cells, skin cells, hair follicle cells, etc. Inhibition of bone marrow cells can lead to neutropenia. The killing of skin cells can lead to the appearance of a rash. Oxaliplatin and CF may also cause allergic reactions, including skin erythema, anaphylactic shock, etc. Allergy usually occurs when oxaliplatin is used for 3 to 5 cycles. The mechanism of this reaction is not yet clear. Some studies believe that allergy occurs when oxaliplatin is given intravenally for 1 hour until a few hours after the end of infusion, which is related to the release of cytokines in vivo. Once allergic reaction occurs, its fatality rate is higher than penicillin, and so careful attention should be paid during its administration. Oxaliplatin intravenous drip, such as insufficient flushing of infusion tube, CF followed by intravenous drip will appear during drug denaturation. Denatured CF binds to proteins in the body and can form complete antigens, causing rapid allergic reactions. Therefore, careless flushing may also give rise to an allergic reaction.

Rash and neutropenia are common adverse reactions of oxaliplatin injection. The common toxicity of $5-\mathrm{FU}$ injection is peripheral leukopenia, whereas thrombocytopenia is rare. Rash, urticaria, asthma, and some other allergic reactions occasionally occur after the administration of CF.

Oxaliplatin in combination with $5-\mathrm{FU}$ and $\mathrm{CF}$ can remarkably improve tumor symptoms while significantly increasing response rates and prolonging progression-free survival (PFS) and time to progression (TTP). However, the incidences of anemia, neutropenia, and thrombocytopenia may also concurrently increase. The most common adverse reactions include digestive disturbances (diarrhea, nausea, vomiting, and mucositis), hematological disorders (neutropenia and thrombocytopenia), and nervous system problems (acute/chronic peripheral neuropathies), which are more common and more serious when these three drugs are used in combination than when used alone.

For neutropenia, pro-granulocyte colony growth factor can be used prophylactically or therapeutically. For rashes, antiallergic treatment should be given before chemotherapy, changes in patients' vital signs should be monitored, and the infusion speed should be strictly controlled. If there is no abnormal reaction, the infusion speed can be increased and finish according to the required time. Symptomatic treatment for rashes includes glucocorticoids, antihistamines, supportive care, etc.

Routine blood test indicated neutropenia before the patient started the fifth round of chemotherapy. After treatment, the neutrophil count returned to normal. During chemotherapy, the patient developed a rash and improved after symptomatic treatment. During the sixth and seventh rounds of chemotherapy, the patient again presented neutropenia and rash. Neutropenia was considered to be an adverse event after FOLFOX treatment in combination with clinical presentation, time correlation, and information from previous studies. During the chemotherapy, the patient has been treated with antiemesis, antiallergic prophylaxis and liver protection. No other adverse reactions occurred except for rash and neutropenia.

For such patients with adverse reactions after 
chemotherapy, it is recommended to give pro-granulocyte colony growth factor and anti-allergic therapy before starting chemotherapy again. During infusion, the infusion speed should be strictly controlled. If there is no abnormal reaction, the infusion speed should be increased and finished within the required time. Oxaliplatin should be rinsed thoroughly after static drip. Patients with rashes were given Glucocorticoids or Antihistamines. The patient's vital signs were observed. If the signs were stable, chemotherapy was continued; if signs are unstable and life-threatening, chemotherapy was stopped.

In our current case, the patient was pretreated with dexamethasone sodium phosphate injection $10 \mathrm{mg}$ iv before chemotherapy, but this failed to prevent allergic reactions. The patient received multiple infusions of FOLFOX treatment and repeatedly experienced rashes and neutropenia. The rash was treated with Methylprednisolone $40 \mathrm{mg}$ iv + Promethazine $25 \mathrm{mg}$ im + Calcium Gluconate $10 \mathrm{~mL}$ iv, and the neutropenia was treated by subcutaneous injection of the recombinant human granulocyte colonystimulating factor injection. The symptoms were relieved, and the treatment was completed. Chemotherapy-induced allergies were considered for the immediate hypersensitivity seen in the patient and for the clinical symptoms, signs, and examination/test findings. As the same allergic reaction had occurred during the previous chemotherapy sessions, changing the chemotherapy drug was considered.

The adverse reactions in our current case might have arisen for the following reasons: (I) the specific batch of the drug might have been defective in some way; (II) the long interval from when the drug was dispensed to when it was administered led to the decomposition of the drug; or (III) the rapid infusion induced patient intolerance. Given the potential causes of adverse reaction, clinicians should carefully evaluate the allergic risks before the initiation of a chemotherapy regimen, and, importantly, any history of allergic reactions should be clarified. Epinephrine, glucocorticoids, and antihistamines must be prepared during chemotherapy. Once neutropenia occurs, symptomatic treatment must be offered before the initiation of a new chemotherapy cycle, and the dispensed chemotherapy drugs must be used immediately. Also, prolonged-infusion dosing can effectively reduce adverse reactions, while patients who suffer from allergic reactions should not use allergyinducing drug(s). Finally, the patient's adverse reactions and changes in vital signs should be closely monitored during chemotherapy, and the chemotherapy regimen should be adjusted or terminated when necessary.

\section{Acknowledgments}

Funding: None.

\section{Footnote}

Reporting Checklist: The authors have completed the CARE reporting checklist. Available at http://dx.doi.org/10.21037/ apm-20-1321

Conflicts of Interest: All authors have completed the ICMJE uniform disclosure form (available at http://dx.doi. org/10.21037/apm-20-1321). The authors have no conflicts of interest to declare.

Ethical Statement: The authors are accountable for all aspects of the work in ensuring that questions related to the accuracy or integrity of any part of the work are appropriately investigated and resolved. The content involved in this case was in accordance with the ethical standards of the institutional and/or national research committee(s) and with the Helsinki Declaration (as revised in 2013). Written informed consent was obtained from the patient for publication of this study and any accompanying images.

Open Access Statement: This is an Open Access article distributed in accordance with the Creative Commons Attribution-NonCommercial-NoDerivs 4.0 International License (CC BY-NC-ND 4.0), which permits the noncommercial replication and distribution of the article with the strict proviso that no changes or edits are made and the original work is properly cited (including links to both the formal publication through the relevant DOI and the license). See: https://creativecommons.org/licenses/by-nc-nd/4.0/.

\section{References}

1. Siegel RL, Miller KD, Jemal A. Cancer statistics, 2015. CA Cancer J Clin 2015;65:5-29.

2. Torre LA, Bray F, Siegel RL, et al. Global cancer statistics, 2012. CA Cancer J Clin 2015;65:87-108.

3. Liu F, Zhao J, Li C, et al. The unique prognostic characteristics of tumor deposits in colorectal cancer patients. Ann Transl Med 2019;7:769.

4. Tsai HL, Huang CW, Ma CJ, et al. An observational study of vascular endothelial growth factor inhibitors as secondline treatment for metastatic colorectal cancer treated 
with bevacizumab plus FOLFIRI beyond progression: the association with RAS mutation and tumor sidedness. Transl Cancer Res 2019;8:2357-70.

5. Mills KT, Bellows CF, Hoffman AE, Kelly TN, Gagliardi G. Diabetes mellitus and colorectal cancer prognosis: a meta-analysis. Dis Colon Rectum 2013;56:1304-19.

6. NCCN Clinical Practice Guidelines in Oncology: Rectal Cancer (2016. V2) [EB/OL]. Available online: http:// guide.medlive.cn/guideline/10843

7. Schmiegel W, Reinacher-Schick A, Arnold D, et al. Capecitabine/irinotecan or capecitabine/oxaliplatin in combination with bevacizumab is effective and safe as firstline therapy for metastatic colorectal cancer: a randomized phase II study of the AIO colorectal study group. Ann Oncol 2013;24:1580-7.

8. Zhang D, Zheng J, Ni M, et al. Comparative efficacy and safety of Chinese herbal injections combined with the FOLFOX regimen for treating gastric cancer in China: a network Meta-analysis. Oncotarget 2017;8:68873-89.

9. Rogers BB, Cuddahy T, Briscella C, et al. Oxaliplatin: Detection and Management of Hypersensitivity Reactions. Clin J Oncol Nurs 2019;23:68-75.

10. Grothey A, Sobrero AF, Shields AF, et al. Duration of Adjuvant Chemotherapy for Stage III Colon Cancer. N Engl J Med 2018;378:1177.

11. Aranda E, García-Alfonso P, Benavides M, et al. First-

Cite this article as: Huang J, Jiang C, Liu Z, Zheng W, Zhu $X$, Huang $Z$. Rash and neutropenia after the administration of oxaliplatin and 5-fluorouracil plus calcium folinate injection: a case report. Ann Palliat Med 2020;9(5):3678-3683. doi: 10.21037/apm20-1321 line mFOLFOX plus cetuximab followed by mFOLFOX plus cetuximab or single-agent cetuximab as maintenance therapy in patients with metastatic colorectal cancer: Phase II randomised MACRO2 TTD study. Eur J Cancer 2018;101:263-72.

12. Pfeiffer P, Sorbye H, Qvortrup C, et al. Maintenance therapy with cetuximab every second week in the firstline treatment of metastatic colorectal cancer (mCRC): The NORDIC-7.5 study by the Nordic Colorectal Cancer Biomodulation Group. Clin Colorectal Cancer 2015;14:170-6.

13. Zhou JC. Practical Oncology. Beijing: People's Medical Publishing House, 1999:340-1.

14. Liu ZY, Wang DC, Zhao J. Experimental study on effects of L-OHP on anti-tumors. The Practical Journal of Cancer 2000;15:151-3.

15. Su YL, Xue Q, Wang WX, et al. Antitumor mechanism of 5 -fluorouracil by quantum chemistry. Central South Pharmacy 2016;(2):142-4.

16. Liu N, Yuan LL, Zhong DQ, et al. Clinical observation of oxaliplatin combined with calcium folinate and 5 -fluorouracil in the treatment of advanced colorectal cancer. Journal of Jining Medical College 2004;27:46-7.

(English Language Editor: J. Gray) 